\title{
ARTICLE
}

\section{Auditory sensory gating in young adolescents with early-onset psychosis: a comparison with attention deficit/hyperactivity disorder}

\author{
Cecilie Koldbæk Lemvigh ${ }^{1,2}$, Jens Richardt Møllegaard Jepsen ${ }^{1,3}$, Birgitte Fagerlund ${ }^{1,2}$, Anne Katrine Pagsberg ${ }^{3,4}$, \\ Birte Yding Glenthøj ${ }^{1,4}$, Jacob Rydkjær ${ }^{1,3}$ and Bob Oranje ${ }^{1,5}$
}

\begin{abstract}
Numerous studies have demonstrated impaired sensory gating in schizophrenia and this impairment has been proposed as a candidate biomarker for the disorder. The typical age of onset for schizophrenia is early adulthood, however a sizable group of patients present with psychotic symptoms before the age of 18 , commonly referred to as early-onset psychosis (EOP). How an earlier onset influences sensory gating is currently unknown. Impaired sensory gating may not be specific to psychosis, but rather a shared disturbance of neurodevelopmental disorders, such as attention deficit/hyperactivity disorder (ADHD). Therefore, the current study investigated P50 suppression in young adolescents (12-17 years old) with either EOP $(N=55)$ or $A D H D(N=28)$ and age and gender matched healthy controls $(\mathrm{HC})(N=71)$. In addition to P50 suppression, N100 and P200 suppression data were also analyzed. No significant group differences in either raw mean P50 amplitude or mean P50 gating ratios were observed between $\mathrm{EOP}, \mathrm{ADHD}$, and HC. Additionally, we observed no P50 suppression deficit in those EOP patients diagnosed with schizophrenia $(N=$ 39). Similarly, we observed no differences in N100 or P200 between the three groups. Healthy levels of P50 suppression were found in both patient groups. The results are in line with some previous studies showing healthy levels of P50 suppression in the early phases of schizophrenia. Our findings do not support P50 sensory gating as a valid biomarker for EOP or ADHD.
\end{abstract}

Neuropsychopharmacology (2020) 45:649-655; https://doi.org/10.1038/s41386-019-0555-9

\section{INTRODUCTION}

Psychotic disorders usually manifest in early adulthood. However, $11-18 \%$ of patients experience their first psychotic episode during childhood or adolescence [1, 2], commonly referred to as earlyonset psychosis (EOP) [3]. Patients with EOP have an increased risk of developing schizophrenia later in life compared to the general population $[4,5]$. In most studies, EOP is associated with a more insidious onset, longer durations of untreated psychosis (DUP), a more severe course of illness and ultimately a poorer prognosis compared to adult-onset psychosis [5-7], albeit some studies find a better prognosis for EOP than hitherto concluded $[1,8]$.

Sensory gating refers to the brain's ability to filter sensory information by reducing responses to repeated exposure to the same sensory stimulus [9]. It is a pre-attentional phenomenon thought to serve as protection against information overload or sensory "flooding" [9]. A widely used method assumed to assess sensory gating is the so-called P50 suppression paradigm. In a typical P50 suppression paradigm, two identical auditory stimuli are presented in close temporal proximity. Healthy subjects show a decreased P50 amplitude in their electroencephalogram (EEG) response to the second stimulus, whereas schizophrenia patients on average show a significantly smaller decrease [10, 11]. In addition, impaired P50 suppression has been demonstrated in antipsychotic-naive, first-episode patients $[12,13]$, unaffected firstdegree relatives, and individuals at high-risk for psychosis [13, 14]. P50 suppression has, therefore, been proposed as a candidate biomarker for schizophrenia [15]. However, there are also studies reporting healthy levels of P50 suppression in patients with schizophrenia, primarily in the early phases of the disease [16-19]. To the best of our knowledge, only one study previously investigated P50 suppression in children with psychosis [20]. The study included patients with childhood-onset schizophrenia (onset prior to age 13). They reported decreased P50 suppression in all patients (age 7-15 years, mean age 10.3 years), indicating that sensory gating is already disturbed during childhood in patients with schizophrenia. However, this study did not include a healthy control group, but instead used previously published P50 ratios from 29 typically developing children (aged 10-15 years), thus not fully covering the age range of the patients. There is evidence suggesting that sensory gating is highly variable in childhood [2123], and it is, therefore, crucial to include an age-matched healthy control group. Moreover, the study only included a small number of patients $(N=10)$, thus limiting the conclusions that can be drawn.

From a potential biomarker point of view, it is also important to investigate whether impaired P50 suppression is specific to

\footnotetext{
${ }^{1}$ Center for Clinical Intervention and Neuropsychiatric Schizophrenia Research (CINS) and Center for Neuropsychiatric Schizophrenia Research (CNSR), Mental Health Centre Glostrup, Glostrup, Denmark; ${ }^{2}$ University of Copenhagen, Faculty of Social Sciences, Institute of Psychology, Copenhagen, Denmark; ${ }^{3}$ Child and Adolescent Mental Health Center,

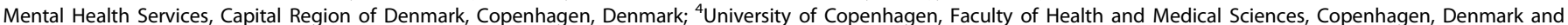
${ }^{5}$ Department of Psychiatry, University Medical Center Utrecht Brain Center, Utrecht University, Utrecht, The Netherlands Correspondence: Cecilie Koldbæk Lemvigh (cecilie.koldbaek.lemvigh@regionh.dk)
}

Received: 9 July 2019 Revised: 23 September 2019 Accepted: 17 October 2019

Published online: 24 October 2019 
psychosis or rather a common deficit among neurodevelopmental disorders, such as attention deficit/hyperactivity disorder (ADHD). Although the clinical symptoms of ADHD differ from schizophrenia, there are common characteristics. Both disorders have been shown to be heritable $[24,25]$ and there is some overlap in early life risk factors $[26,27]$. Moreover, dopamine is thought to be the primary neurotransmitter involved in the pathophysiology of both disorders, although schizophrenia is hypothesized to be associated with a combination of hypo- (frontal areas) and hyperactivity (striatal area) in the dopaminergic system, while ADHD is hypothesized to only involve dopaminergic hypoactivity (frontal areas) $[25,28]$. Compared to the overwhelming literature on adult schizophrenia patients, relatively few studies have investigated P50 suppression in ADHD and the results are inconsistent. Deficient P50 suppression has been demonstrated in adults with ADHD, although to a lesser extent than in schizophrenia [29, 30], yet normal levels of P50 suppression have also been reported [31]. Only one study investigated P50 in children with $A D H D$ and they reported significantly impaired P50 suppression compared to healthy controls [32].

The aim of the present study was to investigate P50 suppression in young adolescents with EOP or ADHD. Based on the literature, we expected the EOP patients to show impaired P50 suppression $[10,11,20]$, with more pronounced deficits in the subgroup of EOP patients who fulfilled the criteria for early-onset schizophrenia (EOS) given that these might represent more severe cases. Additionally, we expected the ADHD patients to show impaired sensory gating, although to a lesser extent than the EOP patients $[30,32]$.

\section{MATERIALS AND METHODS}

The study was approved by the Ethical Committee of the Capital Region of Denmark (H-C-2008-076 \& H-6-2014-068). Informed consent was obtained from parents as well as participants.

\section{Participants}

Patients were recruited from in- and outpatient units at the Child and Adolescent Mental Health Center in the Capital Region of Denmark. Healthy participants were recruited from the local community using internet advertisement and via the Danish Civil Registration System. All participants were between 12 and 17 years old at inclusion. For the present paper, two projects were combined resulting in a sample size of 28 patients with ADHD, 55 patients with EOP and 71 healthy controls $(\mathrm{HC})$. The first project recruited patients with psychosis or ADHD and healthy controls from 2011 to 2014. The subsequent project (2015-2017) was designed as a modified extension study, with no changes in the psychophysiological battery or psychopathological ratings applied but recruiting patients with psychosis and healthy controls only.

In study 1, inclusion criteria for patients with psychosis included a diagnosis of schizophrenia, other non-affective psychosis, or affective psychosis according to DSM-IV-TR criteria [33]. Given that no patients with affective psychosis were actually included for Study 1, Study 2 only included patients with a diagnosis of schizophrenia and other non-affective psychoses. Further inclusion criteria for the EOP group were a score of $\geq 4$ on a minimum of one (or $\geq 3$ on a minimum of two) of the following items of the Positive and Negative Syndrome Scale (PANSS): P1 (delusions), P2 (conceptual disorganisation), P3 (hallucinations), P5 (grandiosity), P6 (suspiciousness/persecution) and G9 (unusual thought content) [34], and a maximum of 12 months cumulative psychopharmacological treatment. Inclusion criteria for the ADHD group were: A diagnosis of ADHD according to DSM-IV-TR [33] and no psychostimulant treatment for the last three months. HC were included if they had no psychiatric illnesses according to DSM-IVTR criteria, no history of psychosis or ADHD in first-degree family members and no ongoing pharmacological treatment (longer than 10-14 days) except for the use of contraceptives.

Exclusion criteria for all groups included: Hearing impairments; A history of neurological illness or significant head injury (loss of consciousness $>5 \mathrm{~min}$ ); and a diagnosis of alcohol or drug dependence according to DSM-IV-TR.

\section{Evaluations of psychopathology}

Somatic examinations were performed to rule out somatic illnesses potentially causing psychiatric symptoms. Diagnoses were assessed using the Kiddie-Schedule for Affective Disorders and Schizophrenia, Present and Lifetime Version (K-SADS-PL) [35]. The same interview was used to screen HCs for psychopathology. Psychotic symptoms were rated with the PANSS interview in all three groups. The level of functioning was rated using the Children's Global Assessment Scale (CGAS) [36] and the Social and Occupational Functioning Assessment Scale (SOFAS) [37]. The assessments were based on consensus ratings between two experienced clinicians, a child and adolescent psychiatrist (J.R.) and a child neuropsychologist (J.R.M.J). The Danish version of the ADHD Rating Scale (ADHD-RS) was completed by the parents [38].

\section{Design}

P50 suppression was assessed as part of the Copenhagen Psychophysiological Test Battery (CPTB), additionally consisting of prepulse inhibition of the startle reflex (PPI), mismatch negativity (MMN) and selective attention paradigms [39, 40], results from which have been (partly) published already [41, 42]. To avoid the acute effects of nicotine and caffeine, all participants were asked to refrain from smoking one hour before testing and from drinking caffeinated beverages two hours before testing. A urine sample was obtained to screen for drug use. Participants were seated in a dimly lit sound-isolated cabin that reduced outside noise with a magnitude of $40 \mathrm{~dB}$ and instructed to sit still with their eyes fixed on a spot on the wall directly in front of them.

\section{P50 paradigm}

The P50 paradigm has been described in detail before [39]. In short, the paradigm consisted of three identical blocks of 40 clickpairs. Each click was $1.5 \mathrm{~ms}$ and had an intensity of $80 \mathrm{~dB}$ (white noise). The interstimulus interval was $500 \mathrm{~ms}$ and all click-pairs were separated by $10 \mathrm{~s}$. Clicks were presented binaurally via stereo insert earphones (Eartone $\mathrm{ABR}, \mathrm{C}$ and $\mathrm{H}$ Distributors Inc., Milwaukee, WI, USA). To avoid drowsiness, participants were instructed to count the number of click-pairs.

\section{Signal processing}

Signal recording and analysis procedures have been described before [39]. BESA software (version 6.0, MEGIS Software GmbH, Gräfelfing, Germany) was used for processing of the EEG data: First, the data was down-sampled from a rate of 2048 to $250 \mathrm{~Hz}$ after which it was corrected for eye-movement by applying the surrogate model of BESA [43]. Then, data were epoched between $100 \mathrm{~ms}$ prestimulus and $400 \mathrm{~ms}$ poststimulus. Thereafter, correction of movement and other non-paradigm related artifacts was performed by removing those epochs from the database that exceeded amplitude differences of $100 \mu \mathrm{V}$ between maximum and minimum in the relevant (between 0 and $120 \mathrm{~ms}$ ) part of the epoch. After averaging, the epochs were band-pass filtered between 1.6 and $70 \mathrm{~Hz}$. P50 amplitudes were obtained from electrode $\mathrm{Cz}$ (with average reference), where maximum P50 amplitude was generally reached in our dataset. P50 amplitude was defined as the largest (preceding) trough to peak amplitude within an interval of $40-90$ ms following the first (conditioning or " $\mathrm{C}^{\prime \prime}$ ) stimulus in each paired click. The P50 amplitude following the second (testing or " $\mathrm{T}$ ") stimulus was identified as the largest (preceding) trough to peak amplitude within an interval of $10 \mathrm{~ms}$ of the latency of the maximum P50 amplitude to the C-stimulus. 
P50 suppression was expressed as the ratio " $T / C$ ", where $T$ is the amplitude to the averaged T-stimuli and $C$ is the amplitude to the averaged $\mathrm{C}$-stimuli. For the interested reader, we also scored the N100 (65-130 ms) and P200 (110-320 ms) waveforms (Supplementary Materials and Methods).

Statistical analysis

All statistical analyses were performed with SPSS (version 22.0, SPSS Inc.). Group differences in gender, age, psychopathology, smoking, and medication status were analyzed with the ChiSquared test or one-way analyses of variance (ANOVA), as appropriate. The PANSS and the ADHD-RS was not normally distributed according to the Kolmogorov-Smirnov test, and was thus analyzed using non-parametric tests. Moreover, neither the P50 raw amplitude data or ratios were normally distributed, however, given the lack of a non-parametric equivalent, the raw P50 amplitude data were analyzed with repeated measures ANOVA, with group as between-factor (ADHD, EOP or HC), and stimulus type as within-factor ( $C$ or $T$ stimulus). The $P 50$ ratio was analyzed using univariate ANOVA to enable inclusion of covariates. We additionally split our EOP group into those patients with and without a schizophrenia diagnosis after which the analyses were repeated. Associations between P50 data and psychopathology rating scales were explored by means of Spearman correlation tests. Similar to the P50 data, the N100 and P200 amplitudes and ratios were not normally distributed, and group differences were analyzed in the same way as the P50 data.

\section{RESULTS}

General

There were no significant differences in age between the three groups, but there were significant differences in gender and smoking status. The EOP patients scored significantly higher on the PANSS positive, negative, general, and total scale compared to both ADHD patients and HC. The ADHD patients scored significantly higher on the ADHD-RS compared to both EOP and HC (Table 1).

Of the 28 patients with ADHD, 23 were diagnosed with the combined type and five with the inattentive type. Of the 55 EOP patients, 39 fulfilled the diagnostic criteria for schizophrenia, six for schizoaffective disorder and 10 were diagnosed with a psychotic disorder not otherwise specified; no patients had an affective psychosis. Thirty-six of the EOP patients were being treated with second-generation antipsychotics at the time of testing. 15 were antipsychotic-naive and four had previously used antipsychotics (one patient had been antipsychotic free for a month, two patients for 3 weeks and the last one up until 2 weeks prior to testing). At the time of testing, none of the ADHD patients were being treated with psychostimulants. Three had previously been treated, yet not within 3 months prior to inclusion.

\section{P50 data}

The average P50 measures are presented in Table 2 . A repeated measures ANOVA on the raw amplitude data revealed a main effect of stimulus type, indicating that the P50 amplitude to Cstimuli was significantly higher than the amplitude in response to T-stimuli $\left[F(1,151)=136.92, p<0.001, \eta^{2}=0.47\right]$ in all three groups. No significant main effect of group, $[F(2,151)=0.45, p=$ $\left.0.63, \eta^{2}=0.01\right]$, nor a significant interaction between stimulus and group were found, $\left[F(2,151)=0.83, p=0.44, \eta^{2}=0.01\right]$ (see Fig. 1). The analysis was repeated with the EOP group split into schizophrenia and non-schizophrenia cases resulting in four groups, which did not change the results significantly.

Analysis of the P50 suppression data (T/C ratio) showed no main effect of group, neither between the three original groups, $\left[F(2,151)=1.37, p=0.26, \eta^{2}=0.018\right]$ nor when split into the four above mentioned groups, $\left[F(3,150)=1.35, p=0.26, \eta^{2}=0.03\right]$.
Adding age, gender and smoking status as covariates in the analyses did not change any of the results significantly, nor did removing participants with a positive urine screening.

Given that more than half of the EOP sample was being treated with antipsychotic medication at the time of testing, we (post-hoc) investigated whether antipsychotic medication had an influence on P50 suppression. We observed no significant differences in P50 ratios between antipsychotic-naive and medicated EOP patients, $(U=232.00, p=0.194, r=-0.18)$.

\section{Correlations between P50 and psychopathology}

In the EOP group, we did not find any significant correlations between either DUP or DUI and P50 suppression. Furthermore, we observed no significant correlations between the P50 measures and the PANSS scale scores in the EOP patients. However, we did find a positive correlation between the T-amplitude and items 10-18 (hyperactivity/impulsivity items) of the ADHD-RS rating scale in this EOP group $\left(r_{s}(50)=0.364, p=0.008\right)$. Similarly, we found a positive correlation between the P50 ratio and items 10-18 on the ADHD-RS (hyperactivity/impulsivity items) $\left(r_{s}(50)=\right.$ $0.367, p=0.007)$. When splitting the EOP group into schizophrenia and non-schizophrenia cases, the observed correlations were no longer significant. In the ADHD group, both the T-amplitude and the $\mathrm{P} 50$ ratio correlated with the PANSS positive score, $r_{\mathrm{s}}(26)=$ $0.412, p=0.029$ and $r_{s}(26)=0.424, p=0.024$, respectively. There were no significant correlations between the P50 data and ratings of psychopathology in the $\mathrm{HC}$ group.

\section{N100 \& P200 data}

No significant group differences were found in either raw amplitude or ratio data on N100 and P200 responses (Supplementary Materials and Methods).

\section{DISCUSSION}

To the best of our knowledge, the current study of P50 suppression included the largest sample of EOP patients to date. Furthermore, this is the first study to directly compare sensory gating between EOP and ADHD.

We found healthy levels of P50 suppression in the EOP patients, which is in contrast with the majority of previous findings from adult patients with schizophrenia $[10,11]$. Given the comparatively large sample size, in combination with the rather weak effectsizes, the negative findings cannot merely be ascribed to power issues. Our EOP findings are in contrast with the single previous study investigating sensory gating in children with schizophrenia, reporting abnormal P50 suppression in all patients [20]. This study by Ross and colleagues [20] only included 10 patients, whereas we included a comparably large group of 55 EOP patients. Furthermore, they only included children with schizophrenia, whereas we included patients with more broadly defined firstepisode psychosis. One may speculate that schizophrenia patients exhibit more pronounced P50 suppression deficits compared to other psychotic disorders. Nevertheless, even those patients in our EOP cohort with a diagnosis of schizophrenia $(N=39)$ did not show P50 suppression deficits. Moreover, the study of Ross and colleagues [20] included patients as young as seven years old. Evidence suggests that P50 suppression matures around the age of 8-10 [22, 23], and it is, therefore possible, that the abnormal P50 suppression observed in their study may, in fact, be an expression of underdeveloped sensory gating. Given our age range (12-17 years old), sensory gating was expected to be fully developed in our participants. Finally, the study of Ross and colleagues [20] defined abnormal P50 suppression as ratios above 0.50 , which may not be an appropriate cut-off, and underscores the importance of including age-matched healthy controls. In a meta-analysis of P50 sensory gating, the mean P50 ratio for healthy controls was found to be 0.39 , ranging from 0.09 to 0.73 
Table 1. Demographics

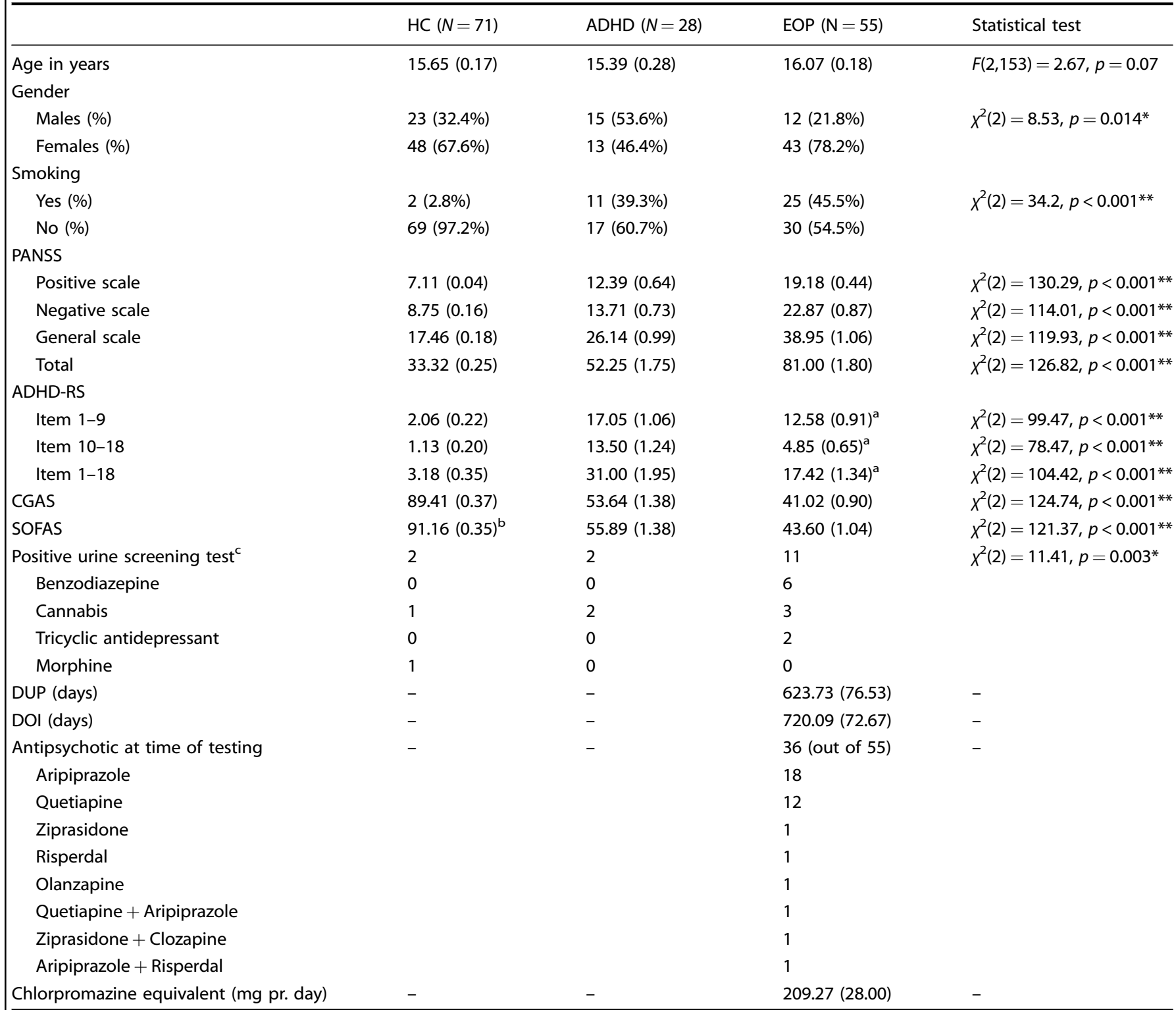

Gender \& smoking status are given in numbers (percentages). The urine screening test and use of antipsychotics are given as number of participants. All other values are mean (SEM)

PANSS The Positive and Negative Syndrome Scale, ADHD-RS The attention deficit/hyperactivity disorder rating scale, CGAS The Children's Global Assessment Scale, SOFAS The Social and Occupational Functioning Assessment Scale, DUP duration of untreated psychosis, DOI Duration of illness

* Significant at the 0.05 level

**Significant at the 0.001 level

${ }^{\text {a }}$ Missing data from 3 EOP patients

${ }^{b}$ Missing data from $3 \mathrm{HC}$

${ }^{c}$ Missing data from four participants (none of which reported frequent use of cannabis or met the criteria for substance dependency)

[11]. The mean P50 gating ratio of our HC group (0.49) is well within this range, thus making abnormal gating in the healthy controls an unlikely argument for the lack of group differences in our study.

Despite the overwhelming evidence demonstrating P50 suppression deficits in schizophrenia patients, there are also studies reporting no such deficits $[17,18]$. We experienced such contrasting findings ourselves in two distinct cohorts of antipsychotic-naive, first-episode adult schizophrenia patients using the same paradigm $[12,16]$, which either raises concerns about the reliability of these findings or suggest heterogeneity in the two datasets. Given that at least two studies have reported high reliability of P50 measures in healthy controls [44, 45], the inconsistent findings more likely point towards the latter; i.e. heterogeneity in schizophrenia, reflected in variability in P50 suppression, at least in the early phases of the disease. Interestingly, this appears not to be the case for other aspects of sensory gating, such as those reflected by prepulse inhibition of the startle reflex (PPI) [16]. Although P50 suppression and PPI are both thought to reflect aspects of sensory gating, results from these paradigms do not correlate [46-48]. In support of this, we previously demonstrated impaired PPI in the first half of our 
Table 2. P50 measures

\begin{tabular}{|c|c|c|c|c|c|}
\hline & $\mathrm{HC}(N=71)$ & $\operatorname{ADHD}(N=28)$ & \multicolumn{3}{|l|}{ EOP } \\
\hline C-amplitude & $2.12(0.14)$ & $1.83(0.23)$ & $2.21(0.21)$ & $2.22(0.25)$ & $2.19(0.38)$ \\
\hline T-amplitude & $0.68(0.08)$ & $0.68(0.13)$ & $0.65(0.09)$ & $0.57(0.11)$ & $0.83(0.16)$ \\
\hline P50 ratio & $0.49(0.10)$ & $0.43(0.09)$ & $0.31(0.04)$ & $0.25(0.05)$ & $0.46(0.09)$ \\
\hline C-latency & $58.37(0.76)$ & $57.64(1.31)$ & $62.51(1.14)$ & $61.69(1.26)$ & $64.50(2.45)$ \\
\hline
\end{tabular}

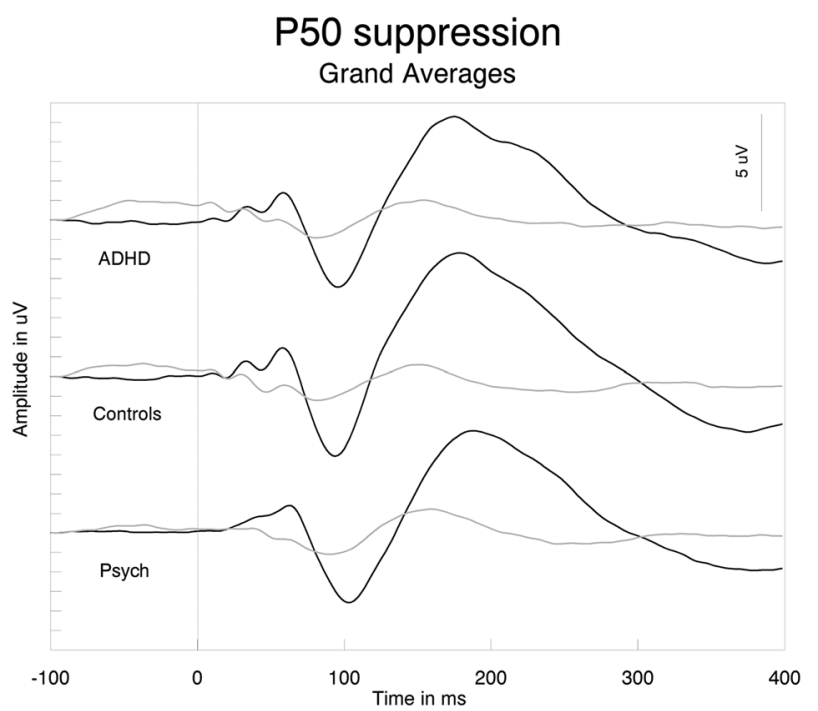

Fig. 1 Grand average data of the P50 waveforms, specified for controls, EOP patients and ADHD patients, showing no significant group differences

current EOP cohort (Study 1) in those EOP patients already diagnosed with schizophrenia [42]. Moreover, we observed impaired MMN in the entire EOP group [41]. These findings suggest that these are not highly atypical patients, given similar findings in previous studies showing deficient MMN and PPI in schizophrenia [49-53].

The primary measure of sensory gating investigated in this study was P50. The suppression of mid-latency auditory evoked responses is thought to include a multistage process. Therefore, we also included analyses of the N100 and P200 waveforms. Here, it is important to realize that previous studies have indicated that N100 suppression is based on refractory mechanisms, rather than pure sensory gating processes, therewith indicating that suppression of the $\mathrm{N} 100$ and P50 amplitudes reflect two different phenomena [54, 55]. Nevertheless, reduced N100 and P200 suppression have been reported in adult patients with schizophrenia $[13,56]$. In line with our P50 data, we failed to find significant group differences in either N100 and P200 amplitudes or ratios between the EOP, ADHD and HC groups.

We included patients with ADHD as a clinical control group to investigate the specificity of P50 suppression. Our finding of healthy levels of P50 suppression in the ADHD group is in line with a previous study of unmedicated adult patients with ADHD [31], but in contrast with those of the sole previous study in children with ADHD [32] as well as two other studies on adult patients with
ADHD $[29,30]$. The negative findings in our study cannot be explained by medication effects given that no psychostimulants were allowed at least three months prior to testing and approximately $90 \%$ of our ADHD sample had never been treated with psychostimulants before. Overall, the inconsistent reports on P50 suppression in ADHD patients suggest heterogeneity in this patient group as well.

In EOP patients, we failed to find a relationship between P50 suppression and psychopathology measured by the PANSS, consistent with a review on clinical correlates of P50 sensory gating [57]. We did find a significant correlation between P50 measures and items 10-18 from the ADHD-RS, indicating that poor P50 suppression is associated with a higher degree of hyperactivity/impulsivity symptoms in EOP. Speculatively, there is an interaction between the psychotic symptoms and symptoms of ADHD in the EOP patients, that causes decreased sensory gating. This may also explain the inconsistent results between studies; if a study is based on a population with high comorbid ADHD symptoms of hyperactivity and impulsivity then P50 suppression is likely decreased (increased P50 ratio) while a study with a population with low comorbid ADHD symptomatology is likely to find no P50 suppression deficits (normal P50 ratio). In the ADHD group, we found a significant correlation between the testing amplitude and the PANSS positive score, indicating that a larger response to the second stimuli, reflective of poor gating, is associated with more subclinical positive symptoms. There are several indicators of overlap between ADHD and schizophrenia that could explain the observed correlations. Higher rates of ADHD have been reported in offspring of schizophrenia patients [58] and attentional deficits in children of parents with schizophrenia has been associated with later development of psychosis [59]. Furthermore, high rates of comorbid ADHD in children and adolescents with schizophrenia has been reported [60, 61].

A major strength of the present study is the inclusion of a clinical control group in addition to healthy controls, thus enabling us to evaluate the diagnostic specificity of P50 suppression. Moreover, we included a relatively large cohort of EOP patients given that these patients are rare and often difficult to recruit. Our cohort contained a four times larger number of schizophrenia patients than that of the sole previous report on P50 suppression and EOS in the literature, making our findings far less susceptible to power issues. The fact that the majority of our ADHD patients were unmedicated might raise concerns about whether the included patients represent milder cases of the disorder. However, the ADHD group scored high on the ADHD-RS, very similar to scores observed in a nationwide multicenter study of children and adolescents with ADHD in Denmark [36], suggesting that these are valid cases. Another potential limitation is the inclusion of medicated EOP patients. Even though we did not detect any effects of antipsychotic 
medication on P50 suppression in the current study it might still have added "noise" to our data that $65 \%$ of the EOP patients were treated with antipsychotics at the time of testing; this, especially given that previous reports indicated that some atypical antipsychotics can normalize P50 suppression deficits in schizophrenia $[12,62]$. One may speculate that the lower average P50 ratio observed in schizophrenia patients $(0.25)$ compared to both non-schizophrenia cases (0.46) and healthy controls (0.49) although not statistically significant, could be explained by schizophrenia patients receiving more antipsychotic medication. However, this do not seem to be the case given that more or less the same percentage of the non-schizophrenia patients as schizophrenia patients were treated with antipsychotics during the time of testing (63 and 67\%, respectively), in spite of the fact that they show such dissimilar average P50 ratios. Moreover, there was no significant difference in the mean chlorpromazine equivalents between the two psychosis groups. More likely the differences are caused by the heterogeneity of our research population, given that the differences did not reach statistical significance. Furthermore we asked participants to count the numbers of clicks to avoid drowsiness. Some studies suggest that mental effort directed at the auditory stimuli may influence sensory gating mechanisms [63-65]. However, the negative findings in the present study are unlikely to be explained by any technical or methodological issues, given that we previously demonstrated P50 suppression deficits in (adult) schizophrenia patients using the same experimental paradigm and instructions $[12,66]$. Finally, this study was cross-sectional, thus limiting the possibility of making causal inferences regarding the effects of maturation and progress of disease.

Summarized, we observed no P50, N100 or P200 amplitude or suppression differences between adolescents with either EOP or ADHD and healthy controls. Based on these results, our study does not supply evidence for sensory gating deficits being a potential biomarker for either EOP or ADHD. Longitudinal studies are needed to examine how sensory gating abilities develop during adolescence and interacts with the initial onset and later progress of psychosis.

\section{FUNDING AND DISCLOSURE}

This work was supported by a PhD and post.doc. scholarship from the Research Fund of the Mental Health Services, Capital Region of Denmark; Lundbeck Foundation Centre of Excellence for Clinical Intervention and Neuropsychiatric Schizophrenia Research (CINS) (grant number R25-A2701); Læge Gerhard Linds legat; Fru C. Hermansens legat; Slagtermester Wörzners og Hustru Inger Wörzners mindelegat, Psykiatrisk Forskningsfond af 1967 and the Jascha Foundation. Dr. Glenthøj is the leader of a Lundbeck Foundation Centre of Excellence for Clinical Intervention and Neuropsychiatric Schizophrenia Research (CINS), which is partially financed by an independent grant from the Lundbeck Foundation based on international review and partially financed by the Mental Health Services in the Capital Region of Denmark, the University of Copenhagen, and other foundations. Her group has also received a research grant from Lundbeck $A / S$ for another independent investigator initiated study. All grants are the property of the Mental Health Services in the Capital Region of Denmark and administrated by them. She has no other conflicts to disclose. The remaining authors declare no competing interests.

\section{ACKNOWLEDGEMENTS}

The authors would like to thank research assistants Gitte Saltoft Andersen (CRN), Mikkel Erlang Sørensen (MSc) and Katharina Alfsen (CRN) and students Rókur av Fløtum Jespersen, Nanna Pagsberg and Andreas Elleby from the Center for Neuropsychiatric Schizophrenia Research (CNSR) for assistance with neurophysiological assessments and registration of data.

\section{ADDITIONAL INFORMATION}

Supplementary Information accompanies this paper at (https://doi.org/10.1038/ s41386-019-0555-9).

Publisher's note Springer Nature remains neutral with regard to jurisdictional claims in published maps and institutional affiliations.

\section{REFERENCES}

1. Amminger GP, Henry LP, Harrigan SM, Harris MG, Alvarez-Jimenez M, Herrman $H$, et al. Outcome in early-onset schizophrenia revisited: findings from the early psychosis prevention and intervention centre long-term follow-up study. Schizophr Res 2011;131:112-9.

2. Schimmelmann BG, Conus P, Cotton S, McGorry PD, Lambert M. Pre-treatment, baseline, and outcome differences between early-onset and adult-onset psychosis in an epidemiological cohort of 636 first-episode patients. Schizophr Res 2007;95:1-8.

3. Werry JS. Child and Adolescent (Early Onset) Schizophrenia: A review in light of DSM-III-R. J Autism Dev Disord. 1992;22:601-24.

4. Jepsen JRM, Fagerlund B, Pagsberg AK, Christensen AMR, Nordentoft M, Mortensen EL. Deficient maturation of aspects of attention and executive functions in early onset schizophrenia. Eur Child Adolesc Psychiatry. 2010;19:773-86.

5. Hollis C. Adult outcomes of child- and adolescent-onset schizophrenia: Diagnostic stability and predictive validity. Am J Psychiatry. 2000;157:1652-9.

6. Ballageer T, Malla A, Manchanda R, Takhar J, Haricharan R. Is adolescent-onset first-episode psychosis different from adult onset? J Am Acad Child Adolesc Psychiatry. 2005;44:782-9.

7. Joa I, Johannessen JO, Langeveld J, Friis S, Melle I, Opjordsmoen S, et al. Baseline profiles of adolescent vs. adult-onset first-episode psychosis in an early detection program. Acta Psychiatr Scand. 2009;119:494-500.

8. Immonen J, Jääskeläinen $\mathrm{E}$, Korpela $\mathrm{H}$, Miettunen J. Age at onset and the outcomes of schizophrenia: a systematic review and meta-analysis. Early Inter Psychiatry. 2017;11:453-60.

9. Adler LE, Pachtman E, Franks RD, Pecevich M, Waldo M, Freedman R. Neurophysiological evidence for a defect in neuronal mechanisms involved in sensory gating in Schizophrenia. Biol Psychiatry 1982;17:639-54.

10. Bramon E, Rabe-Hesketh S, Sham P, Murray RM, Frangou S. Meta-analysis of the P300 and P50 waveforms in schizophrenia. Schizophr Res 2004;70:315-29.

11. Patterson JV, Hetrick WP, Boutros NN, Jin Y, Sandman C, Stern H, et al. P50 sensory gating ratios in schizophrenics and controls: a review and data analysis. Psychiatry Res 2008;158:226-47.

12. Oranje B, Aggernaes B, Rasmussen H, Ebdrup BH, Glenthøj BY. P50 suppression and its neural generators in antipsychotic-Naïve first-episode schizophrenia before and after 6 months of quetiapine treatment. Schizophr Bull. 2013;39:472-80.

13. Brockhaus-Dumke A, Schultze-Lutter F, Mueller R, Tendolkar I, Bechdolf A, Pukrop $\mathrm{R}$, et al. Sensory Gating in Schizophrenia: P50 and N100 Gating in Antipsychoticfree subjects at risk, first-episode, and chronic patients. Biol Psychiatry 2008;64:376-84.

14. Earls HA, Curran T, Mittal V. A meta-analytic review of auditory event-related potential components as endophenotypes for schizophrenia: perspectives from first-degree relatives. Schizophr Bull 2016;42:1504-16.

15. Braff DL, Light GA. The use of neurophysiological endophenotypes to understand the genetic basis of schizophrenia. Dialogues Clin Neurosci. 2005;7:125-35.

16. Düring S, Glenthøj BY, Andersen GS, Oranje B. Effects of dopamine D2/D3 blockade on human sensory and sensorimotor gating in initially antipsychoticnaive, first-episode schizophrenia patients. Neuropsychopharmacology 2014;39:3000-8.

17. De Wilde OM, Bour LJ, Dingemans PM, Koelman JHTM, Linszen DH. Failure to find P50 suppression deficits in young first-episode patients with schizophrenia and clinically unaffected siblings. Schizophr Bull 2007;33:1319-23.

18. Arnfred SM, Chen ACN, Glenthøj BY, Hemmingsen RP. Normal P50 gating in unmedicated schizophrenia outpatients. Am J Psychiatry. 2003;160:2236-8.

19. Morales-Muñoz I, Jurado-Barba R, Fernández-Guinea $S$, Rodríguez-Jiménez R, Jiménez-Arriero MÁ, Criado JR, et al. Sensory Gating Deficits in First-Episode Psychosis. Evidence From Neurophysiology, Psychophysiology, and Neuropsychology. J Nerv Ment Dis. 2016;204:877-84.

20. Ross RG, Olincy A, Harris JG, Radant A, Hawkins M, Adler LE, et al. Evidence for bilineal inheritance of physiological indicators of risk in childhood-onset schizophrenia. Am J Med Genet - Neuropsychiatr Genet. 1999;88:188-99.

21. Freedman R, Adler LE, Waldo M. Gating of the Auditory Evoked Potential in Children and Adults. Psychophysiology 1987;24:223-7.

22. Myles-Worsley M, Coon H, Byerley W, Waldo M, Young D, Freedman R. Developmental and genetic influences on the P50 sensory gating phenotype. Biol Psychiatry 1996;39:289-95. 
23. Brinkman MJR, Stauder JEA. Development and gender in the P50 paradigm. Clin Neurophysiol 2007;118:1517-24.

24. Hilker R, Helenius D, Fagerlund B, Skytthe A, Christensen K, Werge TM, et al. Heritability of schizophrenia and schizophrenia spectrum based on the nationwide Danish twin register. Biol Psychiatry 2017;83:492-8.

25. Biederman J, Faraone SV. Attention-deficit hyperactivity disorder. Lancet 2005;366:237-48.

26. Das Banerjee T, Middleton F, Faraone SV. Environmental risk factors for attentiondeficit hyperactivity disorder. Acta Paediatr 2007;96:1269-74.

27. Matheson SL, Shepherd AM, Laurens KR, Carr VJ. A systematic meta-review grading the evidence for non-genetic risk factors and putative antecedents of schizophrenia. Schizophr Res 2011;133:133-42.

28. Howes OD, Kapur S. The dopamine hypothesis of Schizophrenia: Version III - the final common pathway. Schizophr Bull 2009;35:549-62.

29. Holstein DH, Vollenweider FX, Geyer MA, Csomor PA, Belser N, Eich D. Sensory and sensorimotor gating in adult attention-deficit/hyperactivity disorder (ADHD). Psychiatry Res 2013;205:117-26.

30. Micoulaud-Franchi JA, Vaillant F, Lopez R, Peri P, Baillif A, Brandejsky L, et al. Sensory gating in adult with attention-deficit/hyperactivity disorder: Eventevoked potential and perceptual experience reports comparisons with schizophrenia. Biol Psychol 2015;107:16-23.

31. Olincy A, Ross RG, Harris JG, Young DA, McAndrews MA, Cawthra E, et al. The P50 auditory event-evoked potential in adult attention-deficit disorder: comparison with schizophrenia. Biol Psychiatry 2000;47:969-77.

32. Durukan I, Yucel M, Erdem M, Kara K, Oz O, Karaman D, et al. P50 sensory gating in children and adolescents with ADHD and effects of methylphenidate administration on P50 sensory gating. Bull Clin Psychopharmacol. 2011;21:42-48.

33. APA. Diagnostic and Statistical Manual of Mental Disorders, 4th edn, Text Revision. Washington: American Psychiatric Association; 2000.

34. Kay SR, Fiszbein A, Opler LA. The Positive and Negative Syndrome Scale (PANSS) for Schizophrenia. Schizophr Bull 1987;13:261-76.

35. Kaufman J, Birmaher B, Brent D, Rao U, Flynn C, Moreci P, et al. Schedule for Affective Disorders and Schizophrenia for School-Age Children- Present and Lifetime Version (K-SADS-PL): Initial Reliability and Validity Data. J Am Acad Child Adolesc Psychiatry. 1997;36:980-8.

36. Shaffer D, Gould MS, Brasic J, Ambrosini P, Fisher P, Bird H, et al. A children's global assessment scale (CGAS). Arch Gen Psychiatry. 1983;40:1228-31.

37. Morosini PL, Magliano L, Brambilla L, Ugolini S, Pioli R. Development, reliability and acceptability of a new version of the DSM-IV Social and Occupational Functioning Assessment Scale (SOFAS) to assess routine social functioning. Acta Psychiatr Scand. 2000;101:323-9.

38. Szomlaiski N, Dyrborg J, Rasmussen H, Schumann T, Koch SV, Bilenberg N Validity and clinical feasibility of the ADHD rating scale (ADHD-RS) a Danish Nationwide Multicenter Study. Acta Paediatr. 2009;98:397-402.

39. Oranje B, Wienberg M, Glenthoj BY. A single high dose of escitalopram disrupts sensory gating and habituation, but not sensorimotor gating in healthy volunteers. Psychiatry Res 2011;186:431-6.

40. Jensen KS, Oranje B, Wienberg M, Glenthøj BY. The effects of increased serotonergic activity on human sensory gating and its neural generators. Psychopharmacol (Berl). 2008;196:631-41.

41. Rydkjær J, Møllegaard Jepsen JR, Pagsberg AK, Fagerlund B, Glenthøj BY, Oranje B. Mismatch negativity and P3a amplitude in young adolescents with firstepisode psychosis: a comparison with ADHD. Psychol Med 2017;47:377-88.

42. Rydkjaer J, Jepsen JRM, Pagsberg AK, Fagerlund B, Glenthoej BY, Oranje B Do young adolescents with first-episode psychosis or ADHD show sensorimotor gating deficits? Psychol Med. 2019. 2019. https://doi.org/10.1017/S0033291719000412.

43. Berg P, Scherg M. A multiple source approach to the correction of eye artifacts. Electroencephalogr Clin Neurophysiol. 1994;90:229-41.

44. Hall MH, Schulze K, Rijsdijk F, Picchioni M, Ettinger U, Bramon E, et al. Heritability and reliability of P300, P50 and duration mismatch negativity. Behav Genet 2006;36:845-57
45. Dalecki A, Croft RJ, Johnstone SJ. An evaluation of P50 paired-click methodologies. Psychophysiology 2011;48:1692-1700.

46. Schwarzkopf SB, Lamberti JS, Smith DA. Concurrent assessment of acoustic startle and auditory P50 evoked potential measures of sensory inhibition. Biol Psychiatry 1993;33:815-28

47. Brenner CA, Edwards CR, Carroll CA, Kieffaber PD, Hetrick WP. P50 and acoustic startle gating are not related in healthy participants. Psychophysiology 2004;41:702-8

48. Braff DL, Light GA, Swerdlow NR. Prepulse inhibition and P50 suppression are both deficient but not correlated in schizophrenia patients. Biol Psychiatry 2007;61:1204-7.

49. Erickson MA, Ruffle A, Gold JM. A meta-analysis of mismatch negativity in schizophrenia: from clinical risk to disease specificity and progression. Biol Psychiatry 2016;79:980-7.

50. Umbricht $D$, Krljes $S$. Mismatch negativity in schizophrenia: a meta-analysis. Schizophr Res 2005;76:1-23.

51. Jahshan C, Cadenhead KS, Rissling AJ, Kirihara K, Braff DL, Light GA. Automatic sensory information processing abnormalities across the illness course of schizophrenia. Psychol Med 2012;42:85-97.

52. Kumari V, Soni W, Mathews VM, Sharma T. Prepulse inhibition of the startle response in men with schizophrenia. effects of age of onset of illness, symptoms, and medication. Arch Gen Psychiatry. 2000;57:609-14.

53. Cadenhead KS. Startle reactivity and prepulse inhibition in prodromal and early psychosis: effects of age, antipsychotics, tobacco and cannabis in a vulnerable population. Psychiatry Res 2011;188:208-16.

54. Oranje B, Geyer MA, Bocker KBE, Leon Kenemans J, Verbaten MN. Prepulse inhibition and P50 suppression: Commonalities and dissociations. Psychiatry Res 2006;143:147-58.

55. Davis H, Mast T, Yoshie N, Zerlin S. The slow response of the human cortex to auditory stimuli: Recovery process. Electroencephalogr Clin Neurophysiol. 1966:21:105-13.

56. Boutros NN, Korzyukov O, Jansen B, Feingold A, Bell M. Sensory gating deficits during the mid-latency phase of information processing in medicated schizophrenia patients. Psychiatry Res 2004;126:203-15.

57. Potter D, Summerfelt A, Gold J, Buchanan RW. Review of clinical correlates of P50 sensory gating abnormalities in patients with schizophrenia. Schizophr Bull 2006;32:692-700.

58. Ellersgaard D, Plessen JK, Jepsen JRM, Spang KS, Hemager N, Klee Burton B, et al. Psychopathology in 7-year-old children with familial high risk of developing schizophrenia spectrum psychosis or bipolar disorder - The Danish High Risk and Resilience Study - VIA 7, a population-based cohort study. World Psychiatry 2018;17:210-9.

59. Erlenmeyer-Kimling L, Rock D, Roberts SA, Janal M, Kestenbaum C, Cornblatt $B$, et al. Attention, memory, and motor skills as childhood predictors of schizophrenia-Related Psychoses: The New York High-Risk Project. Am J Psychiatry. 2000;157:1416-22.

60. Ross RG, Heinlein S, Tregellas $H$. High rates of comorbidity are found in childhood-onset schizophrenia. Schizophr Res 2006;88:90-95.

61. Karatekin C, White T, Bingham C. Shared and nonshared symptoms in youthonset psychosis and ADHD. J Atten Disord. 2010;14:121-31.

62. Light GA, Geyer MA, Clementz BA, Cadenhead KS, Braff DL. Normal P50 suppression in schizophrenia patients treated with atypical antipsychotic medications. Am J Psychiatry. 2000;157:767-71.

63. Guterman Y, Josiassen RC. Sensory gating deviance in schizophrenia in the context of task related effects. Int J Psychophysiol. 1994;18:1-12.

64. White PM, Yee CM. Effects of attentional and stressor manipulations on the P50 gating response. Soc Psychophysiological Res. 1997:34:703-11.

65. Dalecki A, Green AE, Johnstone SJ, Croft RJ. The relevance of attention in schizophrenia P50 paired stimulus studies. Clin Neurophysiol 2016;127:2448-54.

66. Oranje B, Glenthøj BY. Clonidine normalizes levels of P50 Gating in patients with schizophrenia on stable medication. Schizophr Bull. 2014;40:1022-9. 